\title{
BMJ Global Health Innovations in cost-effectiveness analysis that advance equity can expand its use in health policy
}

\author{
Anton L.V. Avanceña (D) , ${ }^{1}$ Lisa A Prosser ${ }^{1,2}$
}

To cite: Avanceña AL.V, Prosser LA. Innovations in cost-effectiveness analysis that advance equity can expand its use in health policy. BMJ Global Health 2022;7:e008140. doi:10.1136/ bmjgh-2021-008140

Handling editor Lei $\mathrm{Si}$

Received 29 November 2021 Accepted 29 January 2022

Check for updates

(C) Author(s) (or their employer(s)) 2022. Re-use permitted under CC BY-NC. No commercial re-use. See rights and permissions. Published by BMJ.

${ }^{1}$ Department of Health Management and Policy, University of Michigan, Ann Arbor, Michigan, USA ${ }^{2}$ Department of Pediatrics, Susan B. Meister Child Health Evaluation and Research Center, University of Michigan, Ann Arbor, Michigan, USA

Correspondence to Mr Anton L.V. Avanceña antonlv@umich.edu

\section{INTRODUCTION}

Cost-effectiveness analysis (CEA) provides a useful framework to systematically assess and compare the expected costs and health benefits of two or more alternatives; it has been used around the world, often in the context of health technology assessments (HTAs), to promote efficiency of health systems. Coupled with a consultative process involving various stakeholders, CEA can help inform resource allocation, approval and coverage of medications and other interventions at the national level and prioritisation of patient populations who are most likely to benefit from an intervention, among other objectives. In a handful of countries including the UK, Australia, Thailand, CEA evidence produced by HTA agencies is used in price negotiation and value-based pricing.

By considering cost-effectiveness evidence in their decisions, payers can identify lowvalue services and save significant resources while improving health outcomes. ${ }^{1}$ Several low-income and middle-income countries (LMICs) use CEA to achieve lower drug and vaccine costs and, increasingly, to design healthcare benefits packages for national health insurance programmes that prioritise cost-effective services. ${ }^{2}{ }^{3}$ In the USA, where CEA evidence is not systematically used in, or is explicitly excluded from, healthcare decision-making, payers waste billions of dollars on low-value care. One study found that Medicare, the single largest payer of health services, could save $\$ 12.9$ billion and produce 270000 more quality-adjusted life years (QALYs) among its beneficiaries by reallocating resources from cost-ineffective to cost-saving interventions; an additional 1.8 million QALYs could be gained if Medicare savings were reinvested in other interventions. ${ }^{4}$ Because opportunity costs or foregone benefits of healthcare spending are typically borne by the most socioeconomically
Summary box

Around the world, cost-effectiveness analysis (CEA) is commonly used to evaluate the costs and health benefits of healthcare and public health interventions.

- While suitable to measure the efficiency of health interventions, conventional CEA does not consider health equity concerns that inform decision-making or resource allocation in health.

- Innovations in CEA, such as equity-informative CEA and alternatives to the quality-adjusted life year, are a step in the right direction and can improve the performance of health systems globally.

- By addressing efficiency and equity concerns concurrently, these innovations can reduce unjust health differences and advance a justice-oriented definition of value in health.

disadvantaged in society, waste in the healthcare system can in fact exacerbate existing health inequities. ${ }^{5}$

\section{CEA AND HEALTH EQUITY}

Despite its strengths, CEA has its limitations. CEA was originally developed to maximise efficiency in healthcare; other equally important goals such as achieving health equity or the elimination of unjust health disparities are excluded. Thus, while an intervention might be considered cost-effective, its costs and benefits may be differentially borne by certain groups in society, and a decisionmaker would be unable to identify these distributional consequences in a conventional CEA. ${ }^{6}$ Conventional CEA also does not provide any information about the tradeoffs between efficiency and equity. While advancing equity may often require more resources, ${ }^{7}$ decision-makers may be willing to pay the price provided they know what they are foregoing. The public, too, supports such decisions; survey research suggests that public will prioritise people who are worst off in 
terms of health or socioeconomics or children compared with adults. ${ }^{8}$ Clearly, equity is one of many components of value in health that conventional CEA overlooks.

There are also limitations surrounding the QALY. As the preferred measure of health benefit in CEAs, QALYs conveniently combine quantity and quality of life, but they can undervalue life-extending treatments among patients whose baseline health-related quality of life is low. For example, QALYs associated with extending the life of a person with a spinal cord injury by 1 year is lower compared with a healthy person $(0.63$ vs 1$) ;{ }^{9}$ additionally, treatments targeted to patients with severe conditions and disabilities are often costlier, further reducing an intervention's efficiency. Health gains in CEA are also treated equally independent of baseline quality of life or whether an intervention is saving lives or treating a transient illness, leading to the common expression 'a QALY is a QALY is a QALY'. ${ }^{10}$ An increase in 0.15 QALYs is the same whether this improvement is accrued among people with severe depression or those in full health.

\section{BUILDING ON THE FOUNDATIONS}

CEA methods, however, are evolving, and we believe innovations in CEA that address its inherent limitations will promote its broader use and improve the performance of health systems globally. For example, equity-informative CEA methods have been developed which help quantify the equity effects and potential trade-offs of health interventions. Our recent systematic review found that the number of equity-informative CEAs is increasing, and they have been used to estimate the cost-effectiveness of interventions for a wide range of diseases in several countries. ${ }^{11}$ We found that at least nine dimensions of health equity, from socioeconomic status to race and ethnicity, have been reflected in equity-informative CEAs to date. These results suggest that equity-informative CEAs have the potential to address various health equity concerns in CEA that several HTA agencies explicitly consider in their decision-making. ${ }^{6}$

LMICs may especially benefit from adopting equityinformative CEAs. Faced with limited budgets and significant burden of communicable and non-communicable diseases that disproportionately affect disadvantaged populations, LMICs need decision-making tools that provide information on efficiency and equity. Methods such as distributional CEA, a type of equity-informative CEA, can quantify the distribution of an intervention's costs and health benefits across the population and evaluate equity and efficiency trade-offs. ${ }^{6}$ Another type of equity-informative CEA called extended CEA considers the financial risk protection afforded by the adoption of a publicly financed health intervention, in addition to the distribution of its health gains and costs across population groups. ${ }^{12}$ Extended CEA has been used to evaluate health policies and interventions such as vaccination programmes, mental health treatment and taxes on tobacco and sugar-sweetened beverages. Incorporating these equity-informative methods into the evaluation process for adopting new interventions or programmes can ensure that health equity is front and centre in the decision-making process.

Another way equity is incorporated in CEA ex post is through equity weighting. Though controversial and fraught with empirical challenges, equity weighting is already being used in some countries through a direct adjustment in the cost-effectiveness threshold used to appraise whether an intervention is cost-effective or not. ${ }^{6}$ In the Netherlands and Norway, thresholds are weighted depending on how much a person's quality-adjusted life expectancy is reduced by their disease-a reflection of their concern for treating severe diseases. ${ }^{13}$ In the UK, the National Institute for Health and Care Excellence uses weighted (ie, higher) thresholds for treatments for rare diseases and end-of-life care. By adjusting thresholds, these countries recognise that more resources might be necessary to improve the health of certain populations to achieve equity. Equity weighting used in practice today are based on the disease status of individuals and not their equity-relevant social characteristics (eg, socioeconomic status), though this can also be done in theory. ${ }^{13}$

To address the limitations of QALYs, new metrics have been devised to accompany or replace QALYs in CEAs. For example, ICER, a value assessment organisation in the USA, has started to include equal value of life years gained in their CEA reports in addition to QALYs. ${ }^{14}$ Unlike QALYs, the equal value of life years gained applies the same value to gains in life years whether you are healthy or living with a condition or disability. Another alternative metric is called health years in total, which dissociates gains in longevity and health-related quality of life. ${ }^{15}$ These novel metrics have their share of advantages and drawbacks, but they overcome criticisms of QALYs, particularly from the disability community who believe that QALYs undervalue their lives. It is essential to recognise that these enhanced CEA approaches will likely be used in the same way that conventional CEA is typically considered (ie, as part of a larger decision-making framework), and that there are related efforts to build equity considerations into the decision-making framework such as justice-enhanced CEA and multicriteria decision analysis.

\section{LOOKING FORWARD}

Several challenges preclude wider use of the innovations we have described. First, few analysts have been exposed to these methods, and the capacity to conduct equity-informative CEAs remains limited. Second, equityinformative CEAs require more data than conventional CEA since population-specific inputs are needed to estimate the distribution of costs and health effects of an intervention. While some inputs may be available in public datasets or published literature, estimates from many sources often cannot be disaggregated by an equity-relevant characteristic because the information 
is not routinely collected or specific groups are underrepresented in research. Finally, for distributional CEA in particular, new measures such as population-level estimates of the inequality aversion parameter are needed to determine the preferred health distribution; ${ }^{6}$ these parameters, however, have not been measured outside of selected high-income settings, as is the case for equity weights. ${ }^{10}$

These challenges are surmountable, and several changes are in place that may increase the application of equity-informative CEAs. Data for health equity analyses are becoming more available through national ${ }^{16}$ and international ${ }^{17}$ efforts. Training on novel CEA approaches is increasing, and professional societies are hosting interest groups on economic evaluation methods for equity. Finally, and perhaps most importantly, there is greater awareness of health equity among the public and decision-makers due to the COVID-19 pandemic and renewed racial justice movements. This awareness can lead to interest in new research methods that advance equity, which public and private funders should cultivate. $^{1819}$

\section{CONCLUSION}

Advancing efficiency and equity concurrently is both urgent and necessary. Inaction on widening health inequities leads to social and economic costs that our policy evaluations rarely consider. Persistent inequities cost hundreds of billions in excess medical care and lost productivity and populations that are overlooked, undertreated or mistreated by the healthcare system are only bound to mistrust it. ${ }^{20}$ In other words, inequity is not only unfair but also contributes to inefficiency in our health systems. Equity-informative CEAs present a promising development in health economic evaluation and can bring us closer to a justice-oriented definition of value.

Contributors ALVA conceptualised the paper, wrote the first draft and reviewed and edited the draft. LAP reviewed and edited the draft and provided supervision. Both authors read and approved final version of the paper.

Funding The writing of this paper was supported in part by a grant from the Robert Wood Johnson Foundation to ALVA (Health Policy Research Scholars, \#75647) and by the Susan B. Meister Child Health Evaluation and Research Center at the University of Michigan. The funders played no role in any aspect of this work.

\section{Competing interests None declared.}

Patient consent for publication Not applicable.

Ethics approval This study does not involve human participants.

Provenance and peer review Not commissioned; internally peer reviewed. Data availability statement No data are available.

Open access This is an open access article distributed in accordance with the Creative Commons Attribution Non Commercial (CC BY-NC 4.0) license, which permits others to distribute, remix, adapt, build upon this work non-commercially, and license their derivative works on different terms, provided the original work is properly cited, appropriate credit is given, any changes made indicated, and the use is non-commercial. See: http://creativecommons.org/licenses/by-nc/4.0/.

\section{ORCID iD}

Anton L.V. Avanceña http://orcid.org/0000-0002-4903-870X

\section{REFERENCES}

1 Pandya A. Adding cost-effectiveness to define low-value care. JAMA 2018;319:1977-8.

2 World Health Organization. Global survey on health technology assessment by National authorities main findings, 2015. Available: https://apps.who.int/iris/handle/10665/349093

3 Leech AA, Kim DD, Cohen JT, et al. Are low and middle-income countries prioritising high-value healthcare interventions? BMJ Glob Health 2020;5:e001850.

4 Chambers JD, Lord J, Cohen JT, et al. Illustrating potential efficiency gains from using cost-effectiveness evidence to reallocate Medicare expenditures. Value Health 2013;16:629-38.

5 Love-Koh J, Cookson R, Claxton K, et al. Estimating social variation in the health effects of changes in health care expenditure. Med Decis Making 2020;40:170-82.

6 Cookson R, Griffin S, Norheim OF, eds. Distributional costeffectiveness analysis. Oxford: Oxford University Press, 2021.

7 Galea S. The price of health equity. JAMA Health Forum 2021;2:e210720

8 Eisenberg D, Freed GL, Davis MM, et al. Valuing health at different ages: evidence from a nationally representative survey in the US. Appl Health Econ Health Policy 2011;9:149-56.

9 Whitehurst DGT, Noonan VK, Dvorak MFS, et al. A review of preference-based health-related quality of life questionnaires in spinal cord injury research. Spinal Cord 2012;50:646-54.

10 Lancsar E, Gu Y, Gyrd-Hansen D, et al. The relative value of different QALY types. J Health Econ 2020;70:102303.

11 Avanceña ALV, Prosser LA. Examining equity effects of health interventions in cost-effectiveness analysis: a systematic review. Value Health 2021;24:136-43.

12 World Health Organization. The world bank. global monitoring report on financial protection in health 2021. Geneva: World Health Organization and International Bank for Reconstruction and Development / The World Bank, 2021.

13 Reckers-Droog V, van Exel J, Brouwer W. Willingness to pay for health-related quality of life gains in relation to disease severity and the age of patients. Value Health $2021 ; 24: 1182-92$.

14 Institute for Clinical and Economic Review. 2020-2023 value assessment framework, 2020. Available: https://icer-review.org/ wp-content/uploads/2019/05/ICER_2020_2023_VAF_013120-4.pdf [Accessed 27 Apr 2020].

15 Basu A, Carlson J, Veenstra D. Health years in total: a new health objective function for cost-effectiveness analysis. Value Health 2020;23:96-103.

16 Satcher Health Leadership Institute. Health equity Tracker. health equity Tracker, 2021. Available: https://healthequitytracker.org [Accessed 16 Dec 2021].

17 World Health Organization. Health equity monitor. health Obs, 2021. Available: https://www.who.int/data/gho/health-equity [Accessed 16 Dec 2021].

18 Cookson R, Mirelman AJ, Griffin S, et al. Using cost-effectiveness analysis to address health equity concerns. Value Health 2017;20:206-12.

19 Brownson RC, Kumanyika SK, Kreuter MW, et al. Implementation science should give higher priority to health equity. Implement Sci 2021;16:28.

20 Turner A. The business case for racial equity, 2016. Available: https://www.wkkf.org/resource-directory/resources/2018/07/ business-case-for-racial-equity [Accessed 13 Jul 2020]. 